Relations industrielles

Industrial Relations

Analyse des conduites de salariés en transition de fin de carrière Le cas de travailleurs et travailleuses en situation d'emploi atypique

Analysis of Employee Behaviours in End-of-Career Transition The Case of Workers in Non-Standard Employment

Análisis de las conductas de asalariados en situación de transición hacia el fin de carrera

El caso de los trabajadores y trabajadoras en situación de empleo atípico

Hélène Zimmermann, Geneviève Fournier et Christine Gauthier

Volume 62, numéro 4, automne 2007

Construction de nouveaux rapports à l'organisation du travail dans les transitions professionnelles

Constructing New Relationships with the Work Organization in Occupational Transitions

URI : https://id.erudit.org/iderudit/016959ar

DOI : https://doi.org/10.7202/016959ar

Aller au sommaire du numéro

Éditeur(s)

Département des relations industrielles de l'Université Laval

ISSN

0034-379X (imprimé)

1703-8138 (numérique)

Découvrir la revue

Citer cet article

Zimmermann, H., Fournier, G. \& Gauthier, C. (2007). Analyse des conduites de salariés en transition de fin de carrière : le cas de travailleurs et travailleuses en situation d'emploi atypique. Relations industrielles / Industrial Relations, 62(4), 740-767. https://doi.org/10.7202/016959ar
Résumé de l'article

Cet article propose une analyse des conduites d'investissement de travailleurs et travailleuses dans les différents domaines de leur existence. S'inspirant de l'approche du parcours de vie (life course), il s'appuie sur une enquête qualitative conduite au Québec auprès de 78 salariés âgés de 45 ans et plus, en situation d'emploi atypique depuis au moins trois ans. Les résultats mettent d'abord en évidence le large éventail des conduites observées. Ils montrent aussi la diversité des rapports à l'âge et à l'avenir professionnel des sujets. La prise en compte de ce regard subjectif porté par les travailleurs sur leur étape de vie et leur situation professionnelle s'avère éclairante pour interpréter la diversité des conduites d'investissement observées. Dans une perspective complémentaire, l'article examine dans quelle mesure ces différentes conduites sont statistiquement associées à quelques variables sociobiographiques et de situation professionnelle.
Tous droits réservés @ Département des relations industrielles de l'Université Laval, 2007
Ce document est protégé par la loi sur le droit d'auteur. L’utilisation des services d'Érudit (y compris la reproduction) est assujettie à sa politique d'utilisation que vous pouvez consulter en ligne.

https://apropos.erudit.org/fr/usagers/politique-dutilisation/ 


\section{Analyse des conduites de salariés en transition de fin de carrière}

\section{Le cas de travailleurs et travailleuses en situation d'emploi atypique}

\section{HéLÈne ZimmermanN \\ GENEVIÈVE FourNiER \\ Christine Gauthier}

Cet article propose une analyse des conduites d'investissement de travailleurs et travailleuses dans les différents domaines de leur existence. S'inspirant de l'approche du parcours de vie (life course), il s'appuie sur une enquête qualitative conduite au Québec auprès de 78 salariés âgés de 45 ans et plus, en situation d'emploi atypique depuis au moins trois ans. Les résultats mettent d'abord en évidence le large éventail des conduites observées. Ils montrent aussi la diversité des rapports à l'âge et à l'avenir professionnel des sujets. La prise en compte de ce regard subjectif porté par les travailleurs sur leur étape de vie et leur situation professionnelle s'avère éclairante pour interpréter la diversité des conduites d'investissement observées. Dans une perspective complémentaire, l'article examine dans quelle mesure ces différentes conduites sont statistiquement associées à quelques variables sociobiographiques et de situation professionnelle.

- Zimmermann, H., professionnelle de recherche associée au Centre de recherche et d'intervention sur l'éducation et la vie au travail (CRIEVAT), Université Laval, Québec, Canada, doctorante en sociologie, Université Lumière-Lyon II, Lyon, France, helene. zimmermann.1@ulaval.ca

- FouRnier, G., professeure titulaire au Département des fondements et pratiques en éducation et directrice du Centre de recherche et d'intervention sur l'éducation et la vie au travail (CRIEVAT), Université Laval, Québec, Canada, genevieve.fournier@fse.ulaval. ca

- Gauthier, C., professionnelle de recherche associée au Centre de recherche et d'intervention sur l'éducation et la vie au travail (CRIEVAT), Université Laval, Québec, Canada, diplômée de maîtrise du Département des relations industrielles de l'Université Laval, christine.gauthier@fse.ulaval.ca 
Depuis une vingtaine d'années, les transformations du système de production et de l'organisation du travail provoquent une fragilisation du lien d'emploi traditionnel, ce que traduit notamment le développement de nouvelles formes d'emploi, dites atypiques (Bélanger, Giles et Murray, 2004). Par emploi atypique, nous entendons, à la suite de Bourhis et Wils (2001), tout emploi caractérisé par l'absence de quatre indicateurs : un lien d'emploi durable, le travail à temps plein, un horaire de travail régulier et un travail accompli chez l'employeur. Il résulte du développement de l'emploi atypique une détérioration des conditions de travail et de vie de nombreux salariés et, parallèlement, une plus grande hétérogénéité et instabilité des parcours professionnels. Par ailleurs, ces changements interviennent dans un contexte de vieillissement de la force de travail dans les sociétés occidentales (OCDE, 2006). Au Canada, l'effet combiné $\mathrm{du}$ vieillissement de la population et du ralentissement de la croissance démographique explique que la proportion de la main-d'œuvre de 50 ans et plus a sensiblement augmenté et représente maintenant le tiers de la population active (Rogers et O'Rourke, 2004). Or, les travaux récents attestent la résonance particulière qu'ont les mutations du marché du travail pour les travailleurs et travailleuses vieillissants. Un pourcentage élevé d'entre eux se trouvent confrontés à des parcours de fin de carrière marqués de ruptures et de disqualifications de toutes sortes et éprouvent des difficultés importantes à conserver leur emploi, à une période de leur vie où la plupart croyaient pourtant leur situation professionnelle assurée (Beatty et Fothergill, 2003).

Ces contraintes auxquelles doivent faire face un nombre croissant de salariés vieillissants sont exacerbées par le fait qu'en raison de leur avancée en âge, ces derniers disposent de moins de temps devant eux pour améliorer leur situation sur le marché du travail. Ainsi, non seulement la qualité de leur vie professionnelle est mise en péril, mais le moment de leur retraite, et leurs conditions de vie une fois qu'ils y seront, sont également incertains (He, Colantonio et Marshall, 2006). Ce contexte difficile de fin de parcours professionnel a des répercussions qui dépassent la sphère professionnelle. Il peut, en effet, mener la personne à un repositionnement en profondeur concernant sa vie au travail et hors travail, ainsi qu'à une redéfinition de ses valeurs et priorités de vie. Il semble qu'il suscite notamment des questionnements importants sur le sens accordé à l'activité de travail par rapport aux autres activités et sur la signification que revêt la transition vers la retraite (Gauthier, Fournier et Zimmermann, 2007).

Comment les travailleurs seniors en situation d'emploi atypique appréhendent-ils leur fin de vie professionnelle et la transition vers la retraite ? Dans quelle mesure leurs conduites en matière d'investissement au travail et hors travail traduisent-elles un désinvestissement progressif à 
l'égard du travail au profit de la vie hors travail ? Quelles représentations ces travailleurs se font-ils de leur avancée en âge et de leur avenir professionnel ? Telles sont les principales questions qui ont guidé la présente étude ${ }^{1}$, qui s'inspire de la tradition de l'approche du parcours de vie (life course). Cet article vise d'abord à décrire la diversité des conduites d'investissement des sphères de vie (travail et hors travail) d'un groupe de 78 salariés âgés de 45 ans et plus, qui connaissent un parcours professionnel jalonné d'emplois atypiques. Il cherche également à explorer le rapport à l'âge et à l'avenir de ces travailleurs et ce faisant, à les situer dans leur propre temporalité biographique. Il s'attache ensuite à analyser dans quelle mesure ce rapport à l'âge et à l'avenir est associé à des conduites d'investissement différentes. Finalement, dans une perspective complémentaire, il examine si les conduites observées sont statistiquement liées aux caractéristiques sociobiographiques des personnes et à leur situation professionnelle.

\section{LA FRAGILISATION DU LIEN D'EMPLOI TRADITIONNEL CHEZ LES TRAVAILLEURS ET TRAVAILLEUSES SENIORS}

\section{Le travail atypique persistant en fin de carrière : une vulnérabilité particulière}

La situation des travailleurs seniors sur le marché du travail s'est grandement détériorée au cours des dernières années (OCDE, 2006). Les privilèges historiquement liés à l'avancée en âge et à l'ancienneté (par ex., promotions, sécurité d'emploi) se raréfient. Plus encore, émergent des phénomènes de "dépréciation des salariés âgés » (Guillemard, 2003) et d'âgisme, i.e. des formes de stéréotypie systématique à leur endroit (Faurie, Fraccaroli et le Blanc, 2007). En témoignent certaines pratiques discriminatoires à l'embauche ou certaines politiques d'entreprises, notamment en matière de formation continue, de promotion et de reconnaissance au travail (Jolivet, 2002). Ainsi, en plus d'être particulièrement touchés par le chômage de longue durée (Dubé et Dionne, 2005; Burnay, 2002), les salariés vieillissants, au Canada du moins, sont davantage soumis que les plus jeunes au travail atypique persistant (Kapsalis et Tourigny, 2004). Or, ces emplois sont généralement assortis d'horaires de travail variables et imprévisibles, de même qu'ils sont habituellement sous-qualifiés et insatisfaisants en regard des aspirations de ces travailleurs (Quadagno et al., 2001). Enfin, l'instabilité du lien d'emploi prive une part importante de ces salariés d'une intégration à une communauté de travail, ce qui amoindrit leur pouvoir de négociation et limite leurs possibilités

1. Cette recherche, réalisée sous la direction de Geneviève Fournier, a été financée par le Conseil de recherches en sciences humaines du Canada (CRSH). 
de bénéficier d'un soutien organisationnel (Virtanen et al., 2003). Cette situation est d'autant plus difficile à vivre que les travailleurs seniors ont généralement connu les «deux mondes industriels» (Veltz, 2000) : le monde industriel classique des Trente Glorieuses, hérité du taylorisme, et celui en développement, fondé sur le modèle en réseau. Dans ce dernier, l'efficacité productive repose davantage sur le savoir individuel et les relations informelles entre les acteurs et les schémas organisationnels hiérarchiques et pyramidaux se trouvent en déclin. Propulsés dans la transition entre l'ancienne société salariale et la nouvelle, ces travailleurs se trouvent ainsi souvent privés de repères identitaires fondamentaux (D'Amours, 2002).

Les études récentes révèlent que le travail atypique persistant peut avoir des impacts particuliers pour les travailleurs en fin de carrière. D'abord, une instabilité d'emploi trop forte à cet âge peut se transformer en départ hâtif, le plus souvent involontaire, du marché du travail, ou en attente passive et résignée de la retraite (Beatty et Fothergill, 2003). Ensuite, ces individus connaissent la plupart du temps une baisse de salaire importante, du fait qu'ils ont habituellement déjà occupé des emplois traditionnels, mieux rémunérés, en plus de faire face à des revenus plus incertains (Chan et Huff Stevens, 2001). Cette double vulnérabilité financière a des effets à court terme, mais aussi à moyen et long termes : il n'est pas rare que ces salariés doivent puiser dans les économies qu'ils avaient accumulées pour leur retraite et renoncer à certains projets de vie. En outre, une baisse des revenus à cet âge revêt parfois une valeur symbolique forte qui peut mener au sentiment de perte de maitrise de l'existence (Quadagno et al., 2001). Finalement, le maintien involontaire des salariés vieillissants dans ce type d'emploi a des impacts importants sur la santé psychologique d'un pourcentage élevé d'entre eux (par ex., stress intense, sentiment d'inutilité sociale, isolement) et, parfois aussi, sur leur santé physique (Rogers et O'Rourke, 2004).

\section{Le travail atypique persistant en fin de carrière et les conduites d'investissement dans les différentes sphères de vie}

Les taux de plus en plus élevés de changements d'emploi observés chez les travailleurs seniors viennent à l'encontre de l'idée selon laquelle les parcours de fin de carrière se caractérisent par un désengagement progressif et volontaire du travail, qui précède un retrait clair et anticipé de la vie active (Crespo, 2005). Or, malgré l'accroissement de ces nouveaux parcours, souvent en dents de scie, voire chaotiques, la variabilité interindividuelle et l'interdépendance des conduites adoptées dans les domaines professionnel, familial et social, ont été peu explorées jusqu'à maintenant dans le cas des travailleurs seniors (Henretta, 2003). Certaines recherches montrent 
néanmoins qu'une partie de ces travailleurs choisissent de se désengager progressivement de la sphère professionnelle (D'Amours, 2002). Toutefois, la manière de vivre ce désengagement est variable. Dans certains cas, il traduit une stratégie de défense qui passe par le choix d'instrumentaliser le travail; cela peut alors être l'occasion d'un engagement accru dans la vie hors travail, lorsque les conditions de vie (par ex., ressources financières) le permettent. Dans d'autres cas, le désengagement est associé à une détérioration de la condition physique ou psychologique, qui renforce la désaffiliation à l'égard du marché du travail (Beatty et Fothergill, 2003).

Il semble également que les contraintes liées à l'instabilité de la vie professionnelle amènent nombre de travailleurs à réduire leur participation à des activités sociales ou citoyennes. Le recentrage sur la vie familiale est alors fréquent, dans un contexte où la famille constitue souvent le principal soutien émotionnel face aux épreuves de la vie professionnelle (Nolan, 2002). Les hommes semblent toutefois faire moins souvent ce choix de conduites que les femmes, surtout si l'instabilité de la fin de carrière est soudaine et imprévue et si l'activité professionnelle constituait le repère central de l'identité personnelle et sociale (Quadagno et al., 2001). Finalement, devant les difficultés vécues en fin de carrière, certains travailleurs parviennent difficilement à s'aménager un espace de liberté en marge de leur activité professionnelle et à s'engager dans des activités qui pourraient combler le vide laissé par un travail sans signification (Burnay, 2002). Évoluant dans un cumul de précarités de tous ordres, dans l'impossibilité d'anticiper l'avenir, même à court terme, ces salariés connaissent souvent simultanément la désaffiliation plus ou moins effective du marché du travail, l'appauvrissement de leurs conditions d'existence et le rétrécissement de leur réseau de sociabilité (Paugam, 2000).

\section{L'ÉCLAIRAGE DE L'APPROCHE DU PARCOURS DE VIE (LIFE COURSE)}

Afin de mieux saisir les conduites d'investissement des travailleurs seniors dans les différents domaines de leur existence, nous nous sommes inspirées de l'approche du parcours de vie (life course). Globalement, cette approche vise à comprendre et à expliquer le déroulement des vies humaines à l'intersection de trois temporalités : le temps biologique des processus développementaux individuels (physiques et psychologiques), le temps historique du contexte social global et le temps biographique des trajectoires individuelles (Lalive d'Épinay, 2005). Elle fait de l'étude des transitions de vie, plus particulièrement des transitions professionnelles, un de ses principaux objets de recherche (Bengtson, Elder et Putney, 2005). 


\section{Une grille d'analyse pour les nouveaux parcours de fin de carrière}

L'approche du parcours de vie propose des pistes d'analyse intéressantes pour comprendre la diversification des parcours professionnels des salariés seniors et leur caractère plus aléatoire. Avant de les présenter brièvement, il est utile de préciser ce qui est entendu par « parcours de vie ». En fait, deux plans d'analyse doivent être distingués. Sur le plan social, les parcours de vie sont des parcours institutionnels, correspondant à des modèles de curriculum. Ils régulent matériellement et symboliquement le déroulement des étapes et des transitions qui jalonnent les vies individuelles, généralement en fonction d'âges spécifiques et des rôles qui leur sont dévolus. Au sein d'une société, ces modèles constituent des références communes. Sur le plan individuel, les parcours de vie sont composés d'un ensemble de trajectoires dans les différentes sphères de l'existence (par ex., famille, éducation, travail, vie sociale), plus ou moins enchevêtrées selon les cas (Settersten, 2003). Les parcours de vie individuels s'inscrivent dans un contexte sociohistorique donné et sont balisés par les structures sociales dans lesquelles les personnes évoluent, sans constituer pour autant une simple réplique des parcours institutionnels : individus et institutions entretiennent une relation interactive. Actuellement, on observe une profonde évolution de cette relation, les institutions ayant une propension moindre à formater les parcours individuels. Affectant l'ensemble des sphères de vie, cette évolution de la relation «individu-institution » est cependant plus nette dans le domaine du travail, particulièrement à l'étape de fin de carrière (Bengtson, Elder et Putney, 2005).

Ainsi, selon cette grille d'analyse, durant l'ère industrielle (période fordiste) dominaient des parcours professionnels largement homogènes (phénomène de standardisation), encadrés par l'État et les organisations de travail (institutionnalisation), rythmés par la succession d'étapes ordonnées et définies en fonction de normes d'âges relativement rigides (chronologisation). Régnait alors la tripartition des temps de vie, selon trois catégories d'âge distinctes : préparation (formation)/activité/retraite (loisirs) (Kohli, 1986). Dans ce contexte, la fin de vie professionnelle était fortement prévisible et associée aux privilèges de l'ancienneté.

La plus grande diversité des parcours professionnels observée depuis l'avènement de la société post-industrielle (post-fordiste), particulièrement perceptible en fin de carrière, est interprétée par plusieurs auteurs comme le signe d'une déstandardisation des carrières individuelles, sous l'effet d'une double tendance à la désinstitutionnalisation (moindre encadrement normatif, érosion des modèles de curriculum) et à la déchronologisation (arasement des seuils d'âge) (Henretta, 2003). La fin de vie professionnelle est désormais affectée par la réversibilité des événements, la multiplication et le brouillage des transitions, le flou des statuts, la déconcordance des temps 
sociaux entre formation, travail et loisirs, l'effritement des garanties liées à l'avancée en âge (Gautié, 2003; Guillemard, 2004)2. Plus « contingents » (Heinz, 2003), les parcours professionnels supposent aussi davantage de «négociations » entre individus et institutions, particulièrement dans le contexte d'emplois atypiques. Le risque de vivre une fin de carrière erratique s'en trouve ainsi accru.

\section{Quelques principes pour analyser les conduites des salariés en transition de fin de carrière}

L'approche du parcours de vie privilégie l'étude des événements, des transitions, des étapes charnières (turning points), ainsi que leur enchaînement (sequencing), leur durée (duration) et le moment (timing) auquel ils surviennent dans la vie des individus (Settersten, 2003). Quatre postulats inspirés de cette approche ont guidé l'analyse des conduites des travailleurs et travailleuses en fin de carrière.

D'abord, le principe d' "agency» renvoie à l'idée selon laquelle les sujets sont appréhendés comme des acteurs qui ne sont pas passifs face aux transitions et aux événements auxquels ils sont confrontés : ils construisent leur parcours professionnel à travers leurs conduites et les choix qui les sous-tendent, ainsi qu'à travers leurs projets (Elder, Kirkpatrick Johnson et Crosnoe, 2003). Toutefois, pour construire ces parcours, ils tiennent compte des environnements sociaux dans lesquels ils évoluent et des contraintes comme des ressources que ces derniers comprennent. $\mathrm{Ce}$ principe est fondamental pour appréhender la déstandardisation des parcours professionnels et la pluralité des conduites qui y sont associées.

Ensuite, une attention privilégiée est accordée à la subjectivité des individus, c'est-à-dire à la façon dont ils perçoivent et vivent les événements et transitions qui jalonnent leur parcours professionnel. Ici, l'âge est appréhendé comme un marqueur complexe et fluide, dont les significations sont sociales, mais aussi individuelles : marqueur historique qui révèle, entre autres, des effets de cohorte, l'âge fait également l'objet de représentations personnelles, variables d'un individu à l'autre, et qui orientent les conduites de chacun (Lalive d'Épinay, 2005). Ainsi, la prise en compte des perceptions qu'a le sujet de son avancée en âge et de l'étape de vie professionnelle à laquelle il est rendu, est indispensable pour éclairer le choix de ses conduites. De fait, une même situation de transition professionnelle comme celle de la fin de carrière est susceptible d'être vécue différemment selon les représentations que s'en font les travailleurs,

2. Comme le souligne Crespo (2005), ces mutations constituent des tendances globales : les périodes historiques ne doivent pas être pensées trop schématiquement. 
notamment celles qui concernent les effets de l'avancée en âge sur l'avenir au travail et hors travail.

Par ailleurs, l'analyse d'une transition ou d'un événement professionnel doit être resituée dans l'ensemble de la temporalité biographique de la personne. Ainsi, les conduites observées en matière d'investissement de la vie au travail et hors travail en fin de carrière, et la re-signification du travail qu'elles traduisent le cas échéant, doivent être appréhendées à la lumière des événements passés du parcours professionnel ainsi que des projets de ces travailleurs (Henretta, 2003).

Enfin, l'analyse d'une transition ou d'un événement professionnel doit tenir compte de l'interdépendance des différentes sphères de vie (travail, famille, loisirs, etc.) (Crosnoe et Elder, 2002). Ainsi, l'étude des conduites des travailleurs seniors en matière d'investissement dans les domaines de l'existence suppose de considérer dans quelle mesure le désengagement à l'égard du travail s'associe à l'existence de projets personnels ou encore en quoi la vie personnelle peut favoriser le maintien d'un investissement élevé au travail. Si ce dernier postulat s'applique aux individus de tout âge, la prise en compte de cette interdépendance est d'autant plus importante que l'on s'attend généralement à l'approche de la retraite, à un repositionnement à l'égard du travail et à une redéfinition de l'investissement dans les différentes sphères de vie (Fournier et Bujold, 2005).

\section{MÉTHODOLOGIE}

\section{La description de l'échantillon}

L'échantillon est composé de 38 hommes (48,7\%) et de 40 femmes $(51,3 \%)$. Concernant l'âge des sujets, 33 d'entre eux sont âgés de 45 à 50 ans $(42,3 \%), 19$ sont âgés de 51 à 55 ans $(24,4 \%)$ et 26 ont 56 ans ou plus $(33,3 \%)$. Les personnes se répartissent à peu près également selon leur niveau de scolarité. Ainsi, 26 d'entre elles sont sans qualification ou possèdent un diplôme de niveau secondaire $(33,3 \%), 25$ ont un diplôme de niveau collégial $(32,1 \%)$ et 27 (34,6 \%), un diplôme de niveau universitaire. Concernant leur situation familiale, un peu plus de la moitié vivent seules $(\mathrm{N}=45,57,7 \%)$, et 33 sont en couple $(42,3 \%)$. Enfin, près d'un sujet sur deux se dit en emploi au moment de l'enquête ( $\mathrm{N}=38,48,7 \%), 34$ mentionnent être sans emploi $(43,6 \%)$ et six se déclarent en formation $(7,7 \%)$.

\section{La démarche expérimentale}

Les sujets, recrutés dans la grande région de Québec, ont été rencontrés en entretien individuel semi-structuré d'environ deux heures et demie. Ils 
devaient occuper des emplois atypiques depuis au moins trois ans et ne pas avoir été en retrait du marché du travail pendant plus d'un an d'affilée. Chaque entretien a été enregistré intégralement. Un peu plus de la moitié des entretiens (41), sélectionnés en fonction de la richesse de leur contenu et de leur représentativité au regard de l'âge, du sexe et du niveau de scolarité des sujets, ont été transcrits mot à mot. Les trente-sept autres ont fait l'objet d'une transcription synthétique au plus près des propos des personnes, à partir de notes prises pendant l'entretien et de la réécoute d'extraits d'entretiens, au besoin.

\section{Le protocole d'entretien}

Le protocole d'entretien visait à appréhender les situations professionnelles récentes des personnes, ainsi qu'à les resituer dans la trajectoire professionnelle et le contexte de vie global des sujets. Ce protocole comprenait quatre parties. Premièrement, il s'agissait de retracer les événements ayant jalonné l'histoire de vie professionnelle de la personne depuis son entrée sur le marché du travail, ainsi que ceux vécus dans sa vie hors travail. Deuxièmement, une attention particulière était portée au parcours professionnel des trois dernières années. Outre le recueil d'informations factuelles relatives aux situations professionnelles vécues (emploi, non emploi, formation) et à leur enchaînement, les sujets étaient invités à faire part de leur expérience subjective de chacun de ces événements. À chaque fois, des questions abordaient le rapport entre la vie au travail et hors travail. Troisièmement, une série de questions permettait aux personnes de jeter un regard réflexif sur leur parcours professionnel des trois dernières années. Enfin, la quatrième partie du protocole portait sur quelques représentations socioprofessionnelles des sujets (par ex., retraite) et leurs projets.

\section{La procédure d'analyse}

La méthode d'analyse de contenu adoptée se situe dans le courant interprétatif de la recherche en éducation (Huberman et Miles, 1991) et s'inspire des étapes d'analyse qualitative proposées par L'Écuyer (1990). Après une étape préliminaire, dite de « lecture flottante », effectuée à partir de 31 entretiens, une deuxième étape, de catégorisation et de classification des thèmes, a permis d'élaborer une première ébauche de la grille d'analyse des conduites d'investissement des sphères de vie, ainsi que des rapports à l'âge et à l'avenir. Une relecture du matériel et l'ajout de 15 autres entretiens nous ont ensuite conduites à nuancer et améliorer la grille d'analyse, afin qu'elle reflète le mieux possible la richesse et la complexité des expériences rapportées par les sujets. À cette étape, trois indicateurs ont permis de distinguer plus nettement les conduites des salariés et ont 
servi de base à l'élaboration de la première typologie : 1) la répartition des investissements dans les différentes sphères de vie, au travail et hors travail ${ }^{3}$; 2) le caractère intentionnel ou contraint des conduites observées et, 3) la place et le sens du travail à cette étape de la vie professionnelle. De même, trois autres indicateurs ont été identifiés pour différencier les rapports à l'âge et à l'avenir des sujets et construire la seconde typologie : 1) le regard qu'ils posent sur leur âge chronologique et la façon dont ils interprètent les expériences qui y sont associées; 2) les représentations de leur intégration au marché du travail et de leur avenir professionnel et, 3) la manière dont ils anticipent leur retraite. À la fin de cette étape, l'ensemble des données pertinentes a pu être regroupé en quatre types de conduites d'investissement des domaines de vie et en trois types de rapports à l'âge et à l'avenir.

Afin de valider les deux typologies, deux analystes ont par la suite classé $^{4}$ de manière indépendante les 78 sujets $^{5}$ et en sont arrivés, après discussion des différences de classification, à un consensus ${ }^{6}$. Cette procédure a permis de nuancer et d'affiner les deux typologies existantes. Enfin, comme le suggère L'Écuyer (1990), des traitements quantitatifs complémentaires ont été réalisés afin d'enrichir la description des catégories et leur interprétation.

\section{RÉSULTATS}

Nous présentons d'abord la typologie des conduites d'investissement des sujets dans les sphères de leur vie, puis celle de leur rapport à l'âge et à l'avenir. Nous analysons ensuite dans quelle mesure ces types de rapport à l'âge et à l'avenir sont associés à des conduites d'investissement différentes, dans la vie au travail et hors travail. Finalement, nous examinons si les différentes conduites d'investissement sont statistiquement liées aux caractéristiques sociobiographiques des travailleurs et à certains aspects de leur situation professionnelle.

3. Par investissement d'une sphère de vie, nous entendons à la fois l'engagement psychologique, soit la centralité de cette sphère dans l'existence, et l'engagement temporel, soit le temps passé à pratiquer des activités dans cette sphère. Par sphère de vie hors travail, nous entendons principalement la vie familiale, les loisirs personnels et la participation à la vie sociale.

4. Conformément à l'approche du parcours de vie, pour effectuer ce classement, les analystes ont replacé les conduites observées dans l'histoire de vie professionnelle de la personne, et, plus particulièrement, au sein du parcours professionnel des trois dernières années.

5. Chaque sujet a été classé dans un seul type de conduites et un seul type de rapport à l'âge et à l'avenir.

6. Le taux d'accord interjuges avant la discussion est de $82,1 \%$ (64/78) pour la typologie des conduites et de $85,9 \%$ (67/78) pour celle des rapports à l'âge et à l'avenir. 


\section{Des investissements diversifiés dans les différentes sphères de vie}

\section{TABLEAU 1}

\section{Extraits d'entretiens illustrant les quatre types de conduites d'investissement des sphères de vie}

\begin{tabular}{|c|c|}
\hline $\begin{array}{l}\text { Type } 1 \\
\text { Vers une nouvelle étape de vie } \\
(\mathrm{N}=23 / 78 ; 29,5 \%)\end{array}$ & $\begin{array}{l}\text { «Ça demeure pour moi important que le travail } \\
\text { soit bien fait mais je veux m'y investir beaucoup } \\
\text { moins. [...] Oui, la job, ça facilite ma vie et ça me } \\
\text { permet de m'épanouir mais je peux m'épanouir } \\
\text { ailleurs aussi : dans mes loisirs, avec mes enfants, } \\
\text { en vivant quotidiennement au jour le jour et en } \\
\text { faisant ce qu'il me plaît au moment où ça me } \\
\text { plaît. » } \\
\text { (Femme, } 45-50 \text { ans, diplômée du collégial) }\end{array}$ \\
\hline $\begin{array}{l}\text { Type } 2 \\
\text { Un investissement accru au } \\
\text { travail } \\
(\mathrm{N}=14 / 78 ; 17,9 \%)\end{array}$ & $\begin{array}{l}\text { «En fait je pense que je n'aurai pas beaucoup } \\
\text { de temps jusqu'à } 60 \text { ans pour les loisirs, les } \\
\text { divertissements. [...] Le travail et la formation } \\
\text { viennent presque tout englober, la petite pointe } \\
\text { de tarte de loisirs se rétrécit. [...] Les activités } \\
\text { sociales, c'est familial. [...] C'est un choix que } \\
\text { j'ai fait, je suis heureux dans ce que j'ai fait } \\
\text { comme choix.» } \\
\text { (Homme, } 56 \text { ans et plus, diplômé du collégial) }\end{array}$ \\
\hline $\begin{array}{l}\text { Type } 3 \\
\text { Le partage du temps } \\
(\mathrm{N}=15 / 78 ; 19,2 \%)\end{array}$ & $\begin{array}{l}\text { «L'hiver pour moi, c'est là que c'est plus familial. } \\
\text { On prend au moins une ou deux soirées par } \\
\text { semaine, les cinq ensemble, pour jaser et voir } \\
\text { si tout le monde est correct. [...] Je donne un } \\
\text { break à ma blonde (épicerie, ménage etc.), je me } \\
\text { transforme en « bonne ménagère ». Je bûche mon } \\
\text { bois [...]. C'est du temps à moi, à nous autres. » } \\
\text { (Homme, } 45-50 \text { ans, diplômé du collégial) }\end{array}$ \\
\hline $\begin{array}{l}\text { Type } 4 \\
\text { Vers un décrochage } \\
\text { socioprofessionnel } \\
(\mathrm{N}=26 / 78 ; 33,3 \%)\end{array}$ & $\begin{array}{l}\text { « Dans le fond, dans tous les aspects de ma vie, les } \\
\text { trois dernières années, il n'y a rien de constructif. } \\
\text { [...] C'est négatif et j'abandonne, volontairement } \\
\text { j'abandonne, je suis épuisé, écœuré, je suis } \\
\text { tanné !!! Et même avoir un poste permanent là, } \\
\text { je ne sais même plus si je le prendrais. » } \\
\text { Homme, } 45-50 \text { ans, diplômé du collégial }\end{array}$ \\
\hline
\end{tabular}

1. Vers une nouvelle étape de vie : une réduction choisie de la place du travail au profit de la vie personnelle et familiale

Ces travailleurs, qui représentent plus du quart de l'échantillon, se distinguent par un désinvestissement progressif de la sphère du travail et, 
conjointement, par un investissement accru dans les autres sphères de vie, en particulier de la vie familiale et des loisirs. Il s'agit ici d'une révision majeure des lieux d'investissement, intentionnellement amorcée en fonction d'un nouveau projet de vie, et très étroitement liée au sentiment d'être en fin de vie professionnelle. Si l'activité de travail suscite toujours de l'intérêt chez ces sujets, elle fait l'objet d'une re-signification: les attentes à son égard sont réévaluées à la baisse et sa dimension instrumentale devient plus importante.

Ces personnes ont généralement connu des expériences décevantes dans leur parcours professionnel des dernières années (par ex., faillite), et, à cette étape-ci de leur vie, elles jugent peu probable une progression ou un changement notable dans le déroulement de leur vie au travail. Ainsi, puisque leur vie professionnelle est, de leur point de vue, largement derrière elles, elles choisissent de puiser davantage dans leur vie hors travail, des sources de réalisation personnelle.

2. Une fin de parcours professionnel caractérisée par un investissement accru au travail

Près d'un travailleur sur cinq se distingue par un investissement renforcé et choisi dans la sphère du travail et la décision de reléguer au second plan les activités dans les autres sphères de sa vie, en particulier, la vie sociale et les loisirs. Le travail est plus que jamais au cœur du parcours de vie de ces personnes. Elles y investissent la majeure partie de leur temps et il constitue un lieu-clé d'épanouissement personnel.

Ces sujets ont le sentiment d'une emprise assez forte sur le déroulement de leur parcours professionnel des dernières années et estiment globalement avoir atteint leurs objectifs de carrière. À l'heure où d'autres s'orientent progressivement vers la retraite, ces travailleurs ont, au contraire, l'impression de s'engager dans une période intense de leur histoire de vie au travail et même de connaître une forme de consécration professionnelle.

3. Le partage du temps en fin de vie professionnelle : un investissement équilibré entre travail et vie personnelle

Une troisième catégorie de travailleurs, rassemblant aussi près d'un sujet sur cinq, a fait le choix d'une répartition égale de ses engagements, tant psychologiques que temporels, entre la vie au travail et la vie hors travail (vie familiale et loisirs principalement). Ces individus se distinguent par une polycentration de leurs activités, c'est-à-dire que la sphère du travail occupe une place importante dans leur vie, mais une place parmi d'autres. De fait, si l'activité professionnelle contribue à leur épanouissement, c'est au même titre que celles réalisées hors travail. À la différence des types de conduites précédents, la fin de carrière n'implique pas ici un réajustement 
des investissements dans les différentes sphères de vie. Au contraire, elle correspond au maintien d'un mode de vie, certes relativement marginal, mais dont la formule a été suffisamment éprouvée pour que ces personnes souhaitent la conserver.

Le choix de telles conduites est indissociable du parcours professionnel des dernières années, plus particulièrement du caractère saisonnier ou à temps partiel des emplois occupés. En effet, que ces travailleurs aient ou non initialement fait le choix d'un tel type d'emploi, ils se sont progressivement approprié le mode de vie qui y est associé et, à cette étape de leur carrière, ils jugent le partage du temps entre leurs différentes activités conforme à leurs attentes et à leurs valeurs.

4. Une fin de vie professionnelle déstructurée : vers un décrochage socioprofessionnel

Une dernière catégorie de travailleurs, la plus importante numériquement, manifeste, en fin de carrière, un désengagement psychologique à l'égard de la sphère professionnelle, sans réinvestissement sensible dans les autres sphères d'activité. Ce désengagement constitue une redéfinition majeure, mais contrainte, de leur rapport au travail. Cette dernière est vécue très amèrement à une étape de leur vie où ils espéraient terminer leur carrière sans encombre et réaliser leurs projets de vie hors travail. C'est, du point de vue de ces personnes, toute leur vie qui se trouve déstructurée et enlisée dans une situation sans issue.

Ces travailleurs s'inscrivent généralement depuis plusieurs années dans des parcours professionnels jalonnés de situations récurrentes et involontaires de chômage ou de maintien dans des emplois disqualifiés. Ce contexte professionnel engendre des vulnérabilités de tous ordres (par ex., financier, relationnel et social) qui viennent anéantir les espoirs de réalisation de projets au travail et hors travail. La difficulté de telles expériences est accrue par le sentiment que la proximité de la fin de carrière amoindrit chaque jour davantage la possibilité de réorienter ou d'améliorer leur parcours professionnel. Si ces sujets, comme ceux du type 1 , redéfinissent radicalement leur rapport au travail, ici, une telle conduite est adoptée dans un état de souffrance psychologique d'autant plus intense que le travail revêt encore, sur le plan des représentations idéales, une grande valeur et qu'il n'y a pas de structuration de projets dans les autres domaines de l'existence.

\section{Hétérogénéité des rapports à l'âge et à l'avenir}

Dans la lignée des principes fondamentaux de l'approche du parcours de vie, la typologie que nous présentons ici nous renseigne sur la façon 
dont les sujets interrogés se positionnent dans leur propre temporalité biographique, c'est-à-dire sur la manière dont ils signifient et situent l'étape de leur parcours, par rapport à leurs expériences passées et leurs anticipations d'avenir.

\section{TABLEAU 2}

\section{Extraits d'entretiens illustrant les trois types de rapport à l'âge et à l'avenir}

\begin{tabular}{|c|c|}
\hline $\begin{array}{l}\text { Type } 1 \\
\text { L'âge comme atout } \\
(\mathrm{N}=21 ; 26,9 \%)\end{array}$ & $\begin{array}{l}\text { «Je suis rendu à un âge où je peux apporter ma } \\
\text { contribution. Je suis capable de le faire physiquement. } \\
\text { J'ai pas mal d'expérience, j'ai du vécu, j'ai du bagage } \\
\text { académique. [...] Je n'ai pas l'impression que je pourrais } \\
\text { prendre ma retraite demain matin mais surtout je ne me } \\
\text { sens pas prêt du tout. » } \\
\text { (Homme, } 56-60 \text { ans, diplômé du collégial) }\end{array}$ \\
\hline $\begin{array}{l}\text { Type } 2 \\
\text { L'âge comme facteur de } \\
\text { renoncement } \\
(\mathrm{N}=28 ; 35,9 \%)\end{array}$ & $\begin{array}{l}\text { «Oui j'aurais aimé faire autre chose mais en même } \\
\text { temps, recommencer à zéro ailleurs dans un autre } \\
\text { domaine complètement différent, non. Moi je me dis } \\
\text { que la retraite s'en vient et on ne bouge pas ça. [...] À } \\
\text { court terme je travaille encore } 4 \text { ans et à moyen terme } \\
\text { dans } 4 \text { ans, c'est la retraite. » } \\
\text { (Femme, 51-55 ans, diplômée du secondaire) }\end{array}$ \\
\hline $\begin{array}{l}\text { Type } 3 \\
\text { L'âge comme handicap } \\
(\mathrm{N}=29 ; 37,2 \%)\end{array}$ & $\begin{array}{l}\text { «La retraite c'est une belle utopie. [...] Il y a comme la } \\
\text { psychose du } 40 \text { ans : } 40 \text { ans tu n'es plus bon, tu es moins } \\
\text { que rien. Ils m'ont remplacé par un jeune de } 28 \text { ans que } \\
\text { mon boss appréciait grandement, alors tu te sens moins } \\
\text { que rien. [...] Là, je suis sorti du marché du travail, ce } \\
\text { n'est pas facile de rentrer maintenant. » } \\
\text { (Homme, } 45-50 \text { ans, diplômé de l'université) }\end{array}$ \\
\hline
\end{tabular}

\section{Trois principaux rapports à l'âge et à l'avenir}

1. L'âge comme atout

Cette catégorie rassemble plus du quart des sujets. Pour ces individus, l'avancée en âge est vécue de manière positive. Ils se sentent dans la « force de l'âge » et entretiennent un rapport confiant à l'égard de leur avenir professionnel. À leurs yeux, l'avancée en âge ne constitue pas une limite à leur maintien en emploi, ni à la progression de leur carrière. Au contraire, ils ont le sentiment que les compétences qu'ils ont développées durant leur parcours sont reconnues, et même, recherchées.

Au fur et à mesure des années, ces individus voient leur position sur le marché du travail constamment renforcée. Ainsi, l'heure de la retraite 
leur apparait lointaine et ils ont souvent l'impression d'être au milieu plutôt qu'en fin de carrière. Comme attendu, ces travailleurs se situent majoritairement $(57,1 \%)$ dans la tranche d'âge la plus jeune de notre population (45-50 ans). Néanmoins, une proportion importante $(42,9 \%)$ a 51 ans ou plus.

\section{L'âge comme facteur de renoncement}

La deuxième catégorie regroupe plus du tiers de notre échantillon. Ces individus considèrent leur avancée en âge comme synonyme d'un renoncement nécessaire à toute évolution de leur carrière ou réorientation de leur vie professionnelle. Ils se sentent engagés dans une pente descendante, la cinquantaine constituant à leurs yeux un seuil critique à partir duquel la carrière a atteint son maximum de progression.

Se considérant en bout de piste en raison de leur âge, ces travailleurs ont ainsi amorcé une sorte de deuil professionnel et se résignent à attendre, généralement assez sereinement, la cessation définitive de leur activité professionnelle. Le moment de la prise de retraite est non seulement anticipé, mais aussi, souvent, concrètement planifié. On constate sans surprise qu'environ un individu sur deux $(53,6 \%)$ est âgé de 56 ans ou plus. Par ailleurs, plus du quart $(28,6 \%)$ de ces travailleurs comptent parmi les plus jeunes de notre échantillon (45-50 ans).

\section{L'âge comme handicap}

Cette dernière catégorie regroupe également plus du tiers des sujets. Ces derniers perçoivent leur avancée en âge comme une menace qui fragilise leur présence sur le marché du travail à court, moyen et long termes. L'âge est associé à des handicaps de tous ordres, souvent cumulés : plus grande vulnérabilité professionnelle, diminution des capacités physiques et cognitives... Cette représentation puise sa source principalement dans la perception qu'ils ont du regard négatif de la société et des organisations à l'égard des personnes de 45 ans et plus.

Convaincues que leur avancée en âge constitue un facteur de risque accru de rupture professionnelle définitive, ces personnes se représentent leur fin de carrière sous le jour de l'incertitude et de l'inquiétude qu'elle engendre. Corrélativement, la perception de la retraite est floue et difficilement concevable pour la majorité de ces individus, qui ne peuvent même pas s'imaginer le moment où ils cesseront leur activité professionnelle. Enfin, soulignons que près de la moitié d'entre eux ne sont âgés que de 45 à 50 ans $(44,8 \%)$ et les effectifs diminuent à mesure que l'âge chronologique croît (respectivement $34,5 \%$ pour les $51-55$ ans et $20,7 \%$, pour les 56 ans et plus). 


\section{Conduites des salariés et rapport à l'âge et à l'avenir}

Le rapport à l'âge et à l'avenir s'est avéré pertinent pour éclairer les différentes conduites d'investissement observées. Comme l'illustre le tableau 3 , ces deux variables sont statistiquement associées $\left(\chi_{(6)}^{2}=71,709\right.$; $p<0,001)$.

D'abord, les sujets qui envisagent leur âge comme un atout sont concentrés parmi ceux dont les conduites traduisent un investissement sans équivoque à l'égard du travail. En effet, ils se retrouvent principalement parmi les individus qui, en fin de carrière, choisissent de s'investir fortement au travail, tout en assumant l'emprise que ce choix implique sur leur vie personnelle $(57,1 \%)$. En outre, un tiers d'entre eux $(33,3 \%)$ ont opté pour le partage du temps, en maintenant des investissements qu'ils estiment équilibrés dans les différentes sphères de leur vie. Aucun des sujets qui entretiennent un tel type de rapport à l'âge et à l'avenir n'adopte de conduites de décrochage socioprofessionnel.

Ensuite, percevoir l'avancée en âge comme un facteur de renoncement, un seuil de non-retour, est surtout associé à la décision de se désinvestir progressivement de la sphère professionnelle au profit de la sphère personnelle, dans le cadre d'une redéfinition de ses objectifs de vie. Ainsi, les individus qui ont un tel rapport à leur âge et à leur avenir sont majoritaires parmi les sujets qui ont amorcé une nouvelle étape de vie $(64,3 \%)$. Par ailleurs, seulement un sujet de cette catégorie choisit d'intensifier son investissement au travail.

Enfin, les sujets qui vivent leur âge comme un handicap et entrevoient leur avenir comme étant floué adoptent principalement, en fin de carrière, des conduites de désengagement psychologique à l'égard du travail, sans réinvestir les autres sphères de vie. Ils se retrouvent ainsi massivement dans le type « vers un décrochage socioprofessionnel» $(75,9 \%)$.

\section{Conduites des salariés, caractéristiques sociobiographiques et situation professionnelle}

Nous avons finalement examiné les liens entre les conduites d'investissement des sujets dans les sphères de leur vie et les variables suivantes : genre, âge, niveau de scolarité, situation familiale, situation d'emploi au moment de l'enquête, degré de certitude à l'égard de l'avenir professionnel à moyen terme (précarité professionnelle) et satisfaction financière (tableau 4).

Concernant les variables sociobiographiques, seul le genre des sujets s'avère statistiquement lié aux conduites d'investissement $\left(\chi_{(3)}^{2}=14,587\right.$; $p<0,01)$. Les femmes se trouvent, dans une plus grande proportion que les hommes, engagées vers une nouvelle étape de vie (45\% vs 13,2\%) 


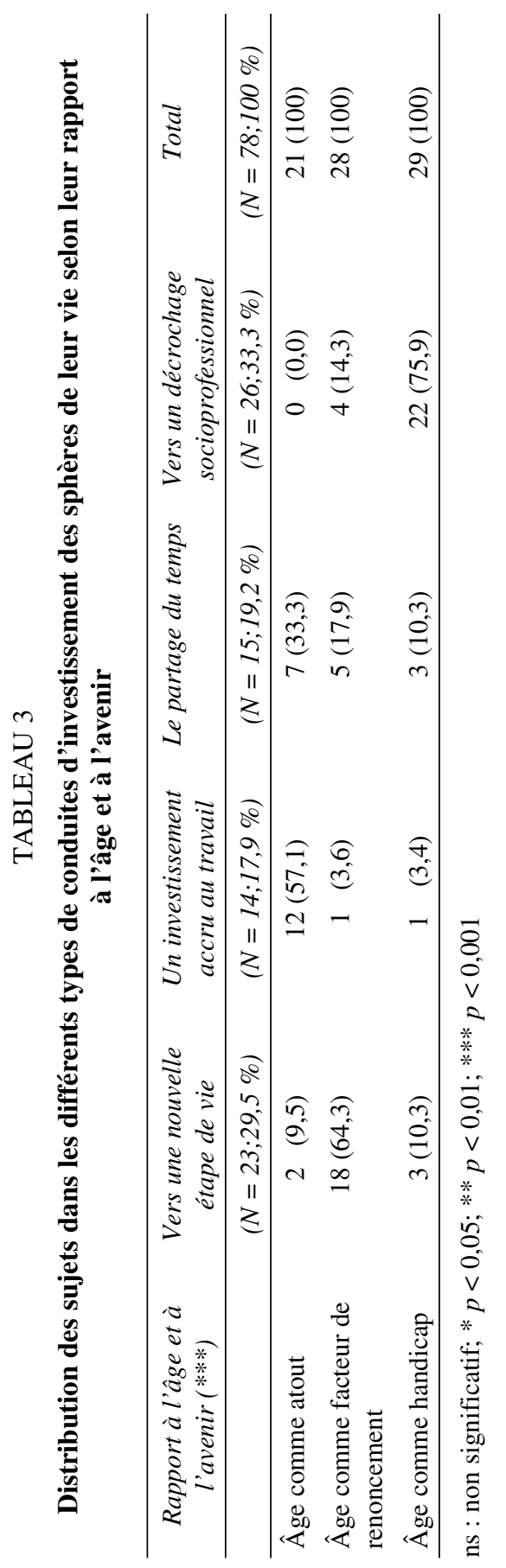




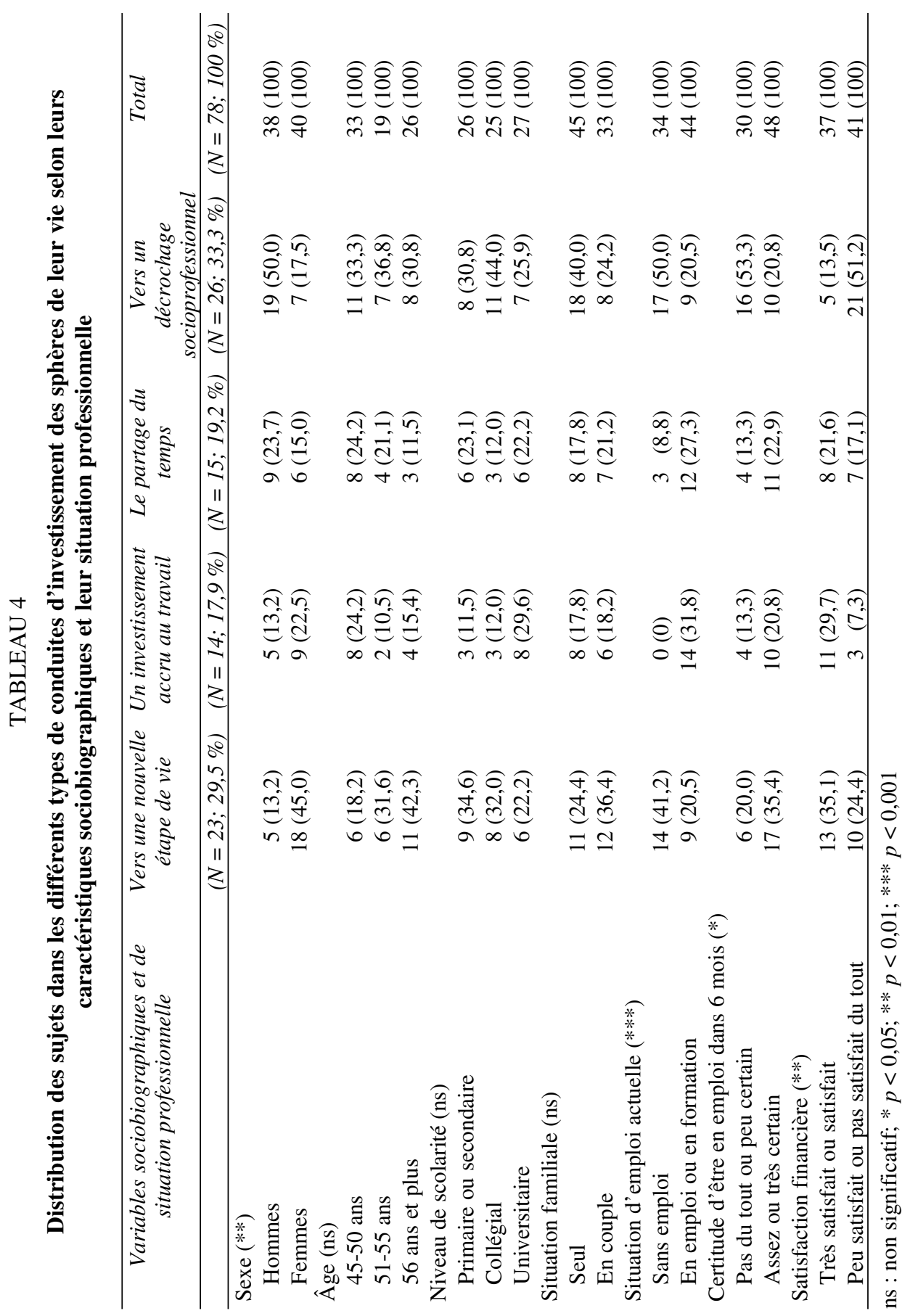


et, dans une moindre mesure, dans un investissement accru au travail $(22,5 \%$ vs 13,2\%). En revanche, les hommes sont surreprésentés dans la conduite de décrochage socioprofessionnel (50\% vs 17,5\%). Aucun lien statistiquement significatif n'est observé avec l'âge chronologique des sujets, leur niveau de scolarité et leur situation familiale. D'ailleurs, concernant l'âge chronologique, les proportions sont très semblables d'une tranche d'âge à l'autre parmi les sujets qui connaissent un décrochage socioprofessionnel $(33,3 \%, 36,8 \%$ et 30,8\%). Cependant, à titre indicatif, on note que les individus de 56 ans et plus s'orientent plus souvent vers une nouvelle étape de vie $(42,3 \%)$ que les plus jeunes $(18,2 \%)$ et que les individus seuls adoptent plus fréquemment des conduites de décrochage socioprofessionnel que ceux qui vivent en couple ( $40 \%$ vs $24,2 \%)$.

Quant aux trois variables de situation professionnelle, toutes sont statistiquement liées aux conduites d'investissement. Ainsi, les travailleurs qui sont sans emploi au moment de l'enquête s'engagent plus souvent dans la voie du décrochage socioprofessionnel (50\% vs 20,5\%) alors que ceux qui sont en emploi ou en formation tendent à s'investir davantage au travail $(31,8 \%$ vs $0 \%)\left(\chi_{(3)}^{2}=22,029 ; p<0,001\right)$. Relativement à la précarité professionnelle $\left(\chi_{(3)}^{2}=8,798 ; p<0,05\right)$, les sujets incertains d'être en emploi dans six mois se trouvent deux fois plus souvent dans des conduites de décrochage socioprofessionnel (53,3\% vs 20,8 \%). Enfin, concernant la satisfaction financière $\left(\chi_{(3)}^{2}=14,709 ; p<0,01\right)$, les personnes insatisfaites sont près de quatre fois plus nombreuses à adopter ces mêmes conduites $(51,2 \%$ vs $13,5 \%)$.

\section{DISCUSSION}

L'objectif du présent article était de décrire les conduites d'investissement dans les différentes sphères de vie de travailleurs et travailleuses seniors en situation d'emploi atypique. Il était aussi de mieux comprendre le rapport à l'âge et à l'avenir de ces personnes puis d'examiner dans quelle mesure les conduites observées étaient associées à ce regard subjectif sur l'avancée en âge. Finalement, dans une perspective d'analyse complémentaire, il s'agissait de savoir si ces conduites étaient statistiquement liées aux caractéristiques sociobiographiques des travailleurs et à leur situation professionnelle.

Les résultats que nous avons présentés suggèrent diverses observations et pistes de réflexion que nous exposons ici succinctement. D'abord, ils ont mis en évidence que le fait de partager la double expérience de l'emploi atypique et de l'avancée en âge engendre des conduites très hétérogènes chez les travailleurs vieillissants. Nous avons ainsi distingué quatre types de conduites d'investissement, qui révèlent autant de manières de réagir 
et de vivre cette situation. Par ailleurs, nous avons discerné trois types de rapports à l'âge et à l'avenir parmi les travailleurs de notre étude. Chaque type de rapport à l'âge et à l'avenir s'est avéré statistiquement associé à des conduites spécifiques.

Globalement, le large éventail des conduites d'investissement rapporté témoigne bien, comme l'a déjà suggéré Gautié (2003), de la plus grande diversité des expériences de fin de carrière et du flou croissant de cette période de transition. Plus spécifiquement, nos résultats montrent que près des deux tiers $(62,8 \%)$ des sujets que nous avons interrogés adoptent globalement des conduites d'éloignement à l'égard du travail et du marché du travail (types 1 et 4). Toutefois, cette réalité regroupe deux catégories d'expériences très distinctes, ce qui peut constituer, selon nous, une illustration partielle de la déstandardisation des parcours de fin de carrière, c'est-à-dire d'une moindre emprise des modèles normatifs sur les parcours individuels (Guillemard, 2004). En effet, seul le type 1 correspond à la conduite normative attendue, selon le schéma traditionnel de tripartition des temps de vie (formation/activité professionnelle/retraite) : le désinvestissement choisi à l'égard du travail, au profit de la vie familiale et des loisirs (Kohli, 1986). Pour les autres sujets (type 4), l'éloignement de la sphère professionnelle est contraint et il n'est pas compensé par des projets hors travail. En outre, à la différence du premier groupe, le travail et la sphère professionnelle constituent toujours, dans l'idéal de ces personnes, le lieu principal de réalisation et de construction identitaire. Le contraste observé entre les types 1 et 4 de conduites suggère aussi que la déstandardisation des parcours de fin de carrière peut constituer une arme à double tranchant : si l'individualisation de ces parcours accrôt la marge de manœuvre et l'autonomie des choix de vie des travailleurs, elle peut aussi les priver de soutien institutionnel et favoriser une fin de carrière plus erratique.

En ce qui concerne l'analyse du rapport à l'âge et à l'avenir des sujets, nos résultats montrent que, pour près des trois quarts d'entre eux $(73,1 \%)$, le regard subjectif porté sur leur avancée en âge est associé au sentiment, plus ou moins prononcé, d'être déprécié sur le marché du travail. Au mieux, ces travailleurs perçoivent leur âge chronologique comme un seuil de non-retour professionnel et s'orientent progressivement vers la retraite. Au pire, ils associent leur âge à des handicaps multiples, compromettant leur présence sur le marché du travail et les conditions de leur retraite. Ce dernier cas nous apparaît préoccupant pour au moins deux raisons. D'abord, ces sujets sont plus nombreux dans la tranche d'âge la plus jeune de notre population (45-50 ans). Ensuite, cette représentation de l'âge comme handicap s'est avérée statistiquement associée au décrochage socioprofessionnel, ce qui laisse présager un risque d'exclusion grandissant au fur et à mesure 
que ces «jeunes» travailleurs avanceront en âge. Ainsi, ce résultat nous semble attester l'existence de ces « demi-vieux» (Guillemard, 2003) qui se retrouvent « ni en emploi, ni vieux » (Réguer, 2007) et, du même coup, d'un vieillissement prématuré d'une partie de nos sujets, notamment ceux qui sont âgés de 45 à 50 ans. Les conséquences en sont à la fois individuelles (expérience douloureuse pour les travailleurs), organisationnelles (perte de ressources humaines expérimentées) et sociales (coûts engendrés par la détérioration des conditions de vie de ces salariés).

Par ailleurs, l'association statistique observée entre les types de conduites d'investissement et l'expérience subjective de l'avancée en âge nous semble confirmer l'intérêt de resituer l'analyse d'un événement ou du choix d'une conduite dans l'ensemble du parcours de la personne, de sa temporalité biographique (Henretta, 2003). Les différences de conduites d'éloignement à l'égard du travail et du marché du travail que nous avons précédemment évoquées (type $1 v s$ 4), illustrent bien ce propos. De fait, ceux qui s'orientent vers une nouvelle étape de vie ont généralement connu une vie au travail qu'ils perçoivent globalement positive et satisfaisante et ce, malgré la déception d'une fin de carrière plus instable qu'ils ne l'avaient planifié. Cependant, ils s'estiment suffisamment valorisés par ce qu'ils ont accompli sur le plan professionnel pour envisager, avec sérénité, de se retirer progressivement, et pour développer des projets. En revanche, la plupart de ceux qui décrochent ont fait l'expérience de parcours professionnels chaotiques ${ }^{7}$, le plus souvent marqués par une ou plusieurs ruptures majeures, qui leur donnent l'impression que leur vie au travail constitue une suite récurrente d'échecs ou de retours à la case départ. Corrélativement, en fin de carrière, ils se sentent floués et dévalorisés professionnellement, de même qu'ils connaissent des précarités multiples auxquelles il leur paraît de plus en plus difficile de faire face, dans l'immédiat, mais aussi dans l'avenir.

Le lien statistique observé entre les types de conduites d'investissement et l'expérience subjective de l'avancée en âge confirme également l'intérêt de considérer la perception qu'ont les travailleurs, non seulement de leur situation professionnelle actuelle, mais aussi de l'étape de vie à laquelle ils sont parvenus et des possibilités d'action qu'ils y associent (Settersten, 2003). De fait, nos résultats révèlent que l'orientation vers une nouvelle étape de vie est statistiquement plus fréquente parmi ceux qui perçoivent leur âge chronologique comme le signal de l'approche de la retraite et un frein à tout nouveau projet professionnel $(64,3 \%)$. En outre, le choix d'un investissement accru au travail se retrouve davantage parmi ceux qui se voient dans la force de l'âge, en pleine progression de carrière et loin de la retraite $(57,1 \%)$, ainsi que, mais dans une moindre mesure, parmi ceux

7. Certains ont cependant connu une stabilité relative dans les débuts de leur carrière. 
qui recherchent un partage équilibré entre leur vie au travail et leur vie hors travail $(33,3 \%)$. Ces derniers perçoivent leur situation professionnelle comme étant remplie d'avantages en regard des objectifs de vie qu'ils poursuivent et l'avancée en âge n'est pas vécue comme un obstacle à l'atteinte de ces objectifs. Quant à la conduite de décrochage, elle concerne principalement les sujets qui associent leur avancée en âge à un cumul de handicaps qui compromettent leur maintien sur le marché du travail, de même que leur avenir professionnel et personnel (75,9\%).

Dans un autre ordre d'idées, nos résultats révèlent que si l'emploi atypique en fin de carrière peut avoir de fortes incidences sur la vie personnelle, réciproquement, cette dernière peut constituer une ressource et influencer l'orientation des conduites au travail. Ainsi, pour les sujets du type 1 , ce sont les projets significatifs hors travail qui facilitent l'amorce d'une transition progressive et relativement sereine vers la cessation définitive d'activité. Pour ceux qui adoptent le partage du temps (type 3), la possibilité d'investir la vie familiale contribue à signifier positivement un emploi saisonnier ou à temps partiel ou encore des sorties plus fréquentes du marché du travail. Quant à ceux qui décrochent (type 4), cette conduite illustre combien une vie professionnelle empreinte de précarités de toutes sortes peut avoir des effets déstabilisants sur l'ensemble des sphères de vie et compromettre les projets au travail et hors travail. Ces résultats viennent confirmer l'importance de prendre en compte les influences réciproques des activités et des projets dans les différentes sphères de vie des sujets pour comprendre leurs choix et leurs conduites, à l'instar de ce qu'ont mis en évidence Crosnoe et Elder (2002).

In fine, nos résultats montrent que, comme l'ont souligné plusieurs tenants de l'approche du parcours de vie, l'analyse du regard subjectif que portent les sujets sur leur situation professionnelle actuelle et sur l'étape de vie à laquelle ils sont parvenus, est essentielle pour appréhender ce qui fonde leurs différents engagements, au travail et hors travail. Ce regard subjectif est étroitement lié à la signification qu'ils attribuent à leur parcours professionnel singulier; ce parcours étant lui-même signifié et interprété en fonction de l'ensemble du parcours biographique. Ainsi, le lien statistique observé entre la diversité de ces conduites et les rapports à l'âge et à l'avenir peut être analysé comme l'expression de l'articulation entre les trois temps biographiques individuels (passé, présent et futur). En effet, d'une part, les conduites des sujets, pour être adéquatement saisies, doivent être resituées dans leur parcours professionnel, au-delà du contexte présent, et rapportées à leur(s) projet(s). D'autre part, les différentes représentations de l'âge et de l'avenir peuvent être lues comme le fruit de la synthèse subjective que chaque travailleur fait des événements professionnels qui jalonnent son parcours ainsi que des contextes personnels et sociétaux qui y sont associés. 
Concernant l'association entre les variables sociobiographiques et les conduites des travailleurs et des travailleuses, peu de résultats significatifs ont été établis. Néanmoins, les femmes décident d'investir davantage leur vie hors travail que les hommes, de même qu'elles adoptent moins souvent des conduites de décrochage socioprofessionnel. Ces résultats concordent avec les études qui montrent que les hommes sont moins enclins à recentrer leurs activités sur la vie familiale (Quadagno et al., 2001). De façon un peu plus surprenante, l'âge chronologique ne s'est révélé associé à aucune conduite particulière. Ceci est particulièrement évident pour la conduite la plus fréquente, le décrochage socioprofessionnel, dont la distribution selon l'âge est pratiquement égale. Ce résultat tend à confirmer la thèse de la déchronologisation des parcours de fin de carrière (de plus en plus évoquée dans les recherches), où l'âge constitue désormais un marqueur fluide, parmi d'autres, plutôt qu'un prescripteur social des conduites (Heinz, 2003). Pour ce qui est de la situation professionnelle des sujets au moment de l'enquête, nos résultats confirment plusieurs associations fréquemment mentionnées dans les publications scientifiques (Beatty et Fothergill, 2003; Chan et Huff Stevens, 2001). Par exemple, les deux indicateurs de la précarité professionnelle (non emploi et incertitude de l'avenir) et l'insatisfaction financière sont associés à des conduites de décrochage socioprofessionnel.

Finalement, notre étude sur les conduites d'investissement des travailleurs vieillissants en situation d'emploi atypique persistant pourrait être prolongée sur plusieurs plans. D'abord, pour valider le lien statistique observé entre les types de conduites d'investissement et certaines variables sociobiographiques et de situation professionnelle, la constitution d'un échantillon plus important de sujets pourrait être pertinente. En particulier, plusieurs associations, non significatives, mais suggérées par les données descriptives, mériteraient d'être testées plus avant. Ainsi en est-il du célibat plus fréquent parmi les travailleurs qui décrochent, alors que la vie de couple semble pouvoir constituer un palliatif à une vie de travail insatisfaisante, ce qui irait dans le sens de l'étude de Nolan (2002). Ensuite, même si l'on peut penser que nos sujets ont intégré le marché du travail dans un contexte relativement semblable et qu'ils ont tous connu les mutations du monde du travail, notre étude repose sur une analyse transversale qui ne permet pas d'exclure tout effet de génération. Un suivi longitudinal des sujets serait donc utile. Il permettrait aussi d'examiner la façon dont l'avancée en âge peut influer sur le sentiment d'être plus particulièrement exposé à des phénomènes d'âgisme. En outre, vu l'importance que revêt l'éloignement de la sphère professionnelle parmi les travailleurs que nous avons étudiés, il serait éclairant d'analyser, dans une perspective comparative, auprès d'un échantillon de travailleurs au parcours de fin de carrière plus « traditionnel » si, d'une part, de telles conduites sont présentes et dans quelles proportions, et si, d'autre part, les significations de l'avancée en âge et de l'avenir sont 
aussi différenciées. Enfin, l'étude présentée dans cet article sera prolongée par des analyses ultérieures des données que nous avons recueillies auprès des travailleurs seniors en emploi atypique persistant. Ces analyses permettront de construire plus finement les parcours biographiques de nos sujets depuis leur entrée sur le marché du travail et d'enrichir la compréhension des liens entre ces parcours et la façon dont est abordée et vécue la fin de carrière.

\section{】 BIBLIOGRAPHIE}

Beatty, Christina et Stephen Fothergill. 2003. «The Over 50s ». Work to Welfare: How Men Become Detached from the Labour Market. P. Alcock, C. Beatty, S. Fothergill, R. McMillan et S. Yeandle, dir. Cambridge: Cambridge University Press, $140-161$.

Bélanger, Jacques, Anthony GiLEs et Gregor Murray. 2004. «Vers un nouveau modèle de production: possibilités, tensions et contradictions ». L'organisation de la production au travail : vers un nouveau modèle. G. Murray, J. Bélanger, A. Giles et P.-A. Lapointe, dir. Québec : Presses de l'Université Laval, 13-62.

Bengtson, Vern L., Glen H. Elder, Jr. et Norella M. Putney. 2005. «The Lifecourse Perspective on Ageing: Linked Lives, Timing, and History ». The Cambridge Handbook of Age and Ageing. M. L. Johnson, V. L. Bengtson, P. G. Coleman et T. B. L. Kirkwood, dir. Cambridge : Cambridge University Press, 493-501.

BouRHIS, Anne et Thierry WILS. 2001. «L'éclatement de l'emploi traditionnel. Les défis posés par la diversité des emplois typiques et atypiques ». Relations industrielles/Industrial Relations, 56 (1), 66-91.

BURNAY, Nathalie. 2002. «Retrait anticipé du marché de l'emploi et exclusion sociale. Le cas des chômeurs âgés en Belgique ». Gérontologie et Société, 102, 109-125.

Chan, Sewin et Ann Huff Stevens. 2001. «The Effects of Job Loss on Older Workers: Employment, Earnings, and Wealth ». Ensuring Health and Income Security for an Aging Workforce. P. P. Budetti, R. V. Burkhauser, J. M. Gregory et H. A. Hunt, dir. Kalamazoo, MI : Upjohn Institute for Employment Research Publications, 189-211.

CRESPO, Stéphane. 2005. Une étude exhaustive des formes de transition vers la retraite. Montréal : INRS-Urbanisation, Culture et Société.

Crosnoe, Robert et Glen H. Elder, Jr. 2002. «Successful Adaptation in the Later Years: A Life Course Approach to Aging ». Social Psychology Quarterly, 65, 309-328.

D'Amours, Martine. 2002. Diversification et fragmentation du travail. Le passage de l'emploi salarié typique à des formes de travail atypique chez les travailleurs de plus de 45 ans. Montréal : INRS-Urbanisation, Culture et Société. 
Dubé, Vincent et Claude Dionne. 2005. « Toujours à la recherche d'un emploi ». L'emploi et le revenu en perspective, 6 (5), 10-15.

Elder, Glen H., Jr., Monica KirKPAtrick Johnson et Robert Crosnoe. 2003. «The Emergence and Development of Life Course Theory ». Handbook of the Life Course. J. T. Mortimer et M. J. Shanahan, dir. New York : Kluwer Academic Publishers, 3-19.

FAurIE, Isabelle, Franco FraCCARoli et Alexis LE BlanC. À paraître. «Âge et travail : des études sur le vieillissement au travail à une approche psychosociale de la fin de la carrière professionnelle ». Le travail humain.

FourniER, Geneviève et Charles Bujold. 2005. « Nonstandard Career Trajectories and their Various Forms ». Journal of Career Assessment, 13, 415-438.

GAUTHIER, Christine, Geneviève FourniER et Hélène ZimMERmann. À paraître. «Le travail atypique persistant des salariés de 45 ans et plus : des expériences plurielles, des choix de vie singuliers ». Psychopathologie du travail et maintien durable en emploi : une question antinomique ? G. Fournier et M.-F. Maranda, dir. Québec : Presses de l'Université Laval.

GAUTIÉ, Jérôme. 2003. « Transitions et trajectoires sur le marché du travail », Quatre Pages $d u$ CEE, $\mathrm{n}^{\circ}$ 59, septembre.

GuILlEMARD, Anne-Marie. 2003. «Âge et marché du travail. Incertitudes de la seconde moitié de carrière ». L'âge de l'emploi : les sociétés à l'épreuve du vieillissement. A.-M. Guillemard, dir. Paris : Armand Colin, 22-59.

Guillemard, Anne-Marie. 2004. «Recomposition des temps de la vie et reconfiguration de la protection sociale ». Recomposition des cycles de vie, réarticulation des temps de la vie, accompagnement des transitions et sécurisation des trajectoires : mutations et politiques. J. Gautié et A.-M. Guillemard, dir. Rapport ACI Travail, Temps, Trajectoires et Transitions, Partie III, Paris : CEE, 31-62.

He, Helen Y., Angela Colantonio et Victor W. Marshall. 2006. « Instabilité professionnelle en fin de carrière et santé après la retraite ». Les nouvelles frontières de recherche au sujet de la retraite. L. O. Stone, dir. Ottawa : Statistique Canada, 351-374.

HeInZ, Walter R. 2003. «From Work Trajectories to Negotiated Careers: The Contingent Work Life Course ». Handbook of the Life Course. J. T. Mortimer et M. J. Shanahan, dir. New York : Kluwer Academic Publishers, 185-204.

Henretta, John C. 2003. «The Life-Course Perspective on Work and Retirement ». Invitation to the Life Course. Toward New Understandings of Later Life. R. A. Settersten, Jr., dir. Amityville, NY : Baywood Publishing Company, 85-105.

Huberman, Michael A. et Matthew B. Miles. 1991. Analyse des données qualitatives : recueil de nouvelles méthodes. Bruxelles : De Boeck.

JoLIVET, Annie. 2002. «Les comportements des entreprises à l'égard des salariés vieillissants ». Gérontologie et Société, 102, 87-107.

KAPSALIS, Costa et Pierre TOURIGNy. 2004. «La durée de l'emploi atypique ». L'emploi et le revenu en perspective, 5 (12), 5-14. 
KoHLI, Martin. 1986. «The World We Forgot: An Historical Review of the Life Course ». Later Life: The Social Psychology of Aging. V. M. Marshall, dir. Beverly Hills, CA : Sage, 271-303.

LALIVE D'ÉPINAY, Christian. 2005. « De l'étude des personnes âgées au paradigme du parcours de vie ». L'analyse du social : les modes d'explication. D. Mercure, dir. Québec : Les Presses de l'Université Laval, 141-167.

L'ÉCUYER, René. 1990. Méthodologie de l'analyse développementale de contenu. Méthode GPS et concept de soi. Québec : Presses de l'Université du Québec.

Nolan, Jane. 2002. « The Intensification of Everyday Life ». Job Insecurity and Work Intensification. B. Burchell, D. Ladipo et F. Wilkinson, dir. London : Taylor \& Francis Group, 92-111.

OCDE. 2006. Vivre et travailler plus longtemps. Paris : OCDE.

Paugam, Serge. 2000. Le salarié de la précarité : les nouvelles formes de l'intégration professionnelle. Paris : Presses universitaires de France.

Quadagno, Jill, David MacPherson, Jennifer ReId KeEne et Lori Parham. 2001. "Downsizing and the Life-Course Consequences of Job Loss: The Effect of Age and Gender on Employment and Income Security ». Restructuring Work and the Life Course, V. W. Marshall, W. R. Heinz, H. Krüger et A. Verma, dir. Toronto : University of Toronto Press, 303-318. RÉGUER, Daniel. 2007. «Introduction ». Vieillissement et parcours de fins de carrière : contraintes et stratégies. D. Réguer, dir. Ramonville Saint-Agne, France : Érès, 11-21.

Rogers, Mary E. et Norm O'Rourke. 2004. « Health, Job Loss and Programs for Older Workers in Canada ». The Canadian Journal of Career Development, 3 (1), 35-42.

Settersten, Richard A., Jr. 2003. "Propositions and Controversies in Life-Course Scholarship ». Invitation to the Life Course: Toward New Understandings of Later Life. R. A. Settersten, Jr., dir. Amityville, NY : Baywood Publishing Company, 15-45.

Veltz, Pierre. 2000. Le nouveau monde industriel. Paris : Gallimard.

Virtanen, Marianna, Mika KivimÄKi, Pekka Virtanen, Marko Elovainio et Jussi VAhteRA. 2003. « Disparity in Occupational Training and Career Planning between Contingent and Permanent Employees ». European Journal of Work and Organizational Psychology, 12 (2), 19-36.

\section{SUMMARY}

\section{Analysis of Employee Behaviours in End-of-Career Transition: The Case of Workers in Non-Standard Employment}

Over the last twenty years, we have witnessed an expansion in nonstandard forms of employment and, as its corollary, more heterogeneous 
and more unstable career trajectories. Older workers, whose numbers in the workforce in Western countries have been growing, have been particularly affected by these changes. These workers have been affected by acute vulnerability in their lives both at work and outside work. Their end-ofcareer trajectories and the transitions associated with them have been less and less certain. This article examines the involvement behaviours of a group of older workers whose recent career trajectory has been based on non-standard jobs. More specifically, the article first describes the types of behaviour observed, then presents the relationship between age and the subjects' future, and then examines whether the latter is associated with these types of behaviour. Lastly, in addition, it assesses whether these behaviours are also statistically linked with the socio-biographical characteristics of the individuals and their employment situation.

The approach adopted is based on the life course perspective which places emphasis on the study of occupational transitions, such as the end-ofcareer transition. From this perspective, the authors selected four principles guiding their study: the worker's status as an actor, capable of making choices and formulating plans; a main focus on the subjectivity of the individuals, in particular their representations of their age and the stage of life they are in; the necessary interpretation of present experiences in terms of one's previous life course and expectations for the future; and the interdependency of the various spheres of life. This approach proves to be particularly useful in the current context of evolving end-of-career trajectories, which can, based on this analytical framework, be interpreted as a triple trend towards disinstitutionalization, dechronologization and destandardization.

This study is based on a corpus of 78 semi-structured interviews conducted in Quebec with men and women aged 45 or older, who have been in non-standard employment for at least three years.

The content analysis brought out four types of involvement behaviours in different spheres of life, at the end-of-career stage: (1) shifting to a new stage of life, characterized by gradual disinvolvement from the work sphere with increased involvement in family life and leisure activities (29.5\%); (2) reinforced and decisive involvement in the work sphere and the decision to relegate non-work projects to the background (17.9\%); (3) choosing an equal distribution of commitments, both psychological and temporal, to both life at work and life outside work (19.2\%); (4) starting a socio-occupational dropping out process, marked by psychological disinvolvement from the work sphere, without significant re-involvement in other spheres (33.3\%). Each of these types of behaviour proved to be associated with experiences specific to the workers' recent career trajectory.

In accordance with the life course approach, a second typology was developed to understand the workers' subjective representation of 
their advancing age and the stage of life they are in, based on their past experiences and their plans for the future. Three distinct types emerged. Thus, some of these workers view their age as a strength which guarantees their labour market retention (26.9\%); some see their age as a social limit holding back any new career plan and get into a position of waiting for retirement (35.9\%); and some think of their age as a source of multiple vulnerabilities and discrimination, which undermines their future at work (37.2\%).

The authors then bring out a statistical link between the relationship with age and the future and the observed types of involvement behaviour. Taking into account this subjective variable proves to be particularly revealing for interpreting the diversity of behaviours. Moreover, the examination of several socio-biographical variables or variables linked to the employment situation at the time of the survey (gender, age, education level, family situation, employment insecurity, financial satisfaction) reveals a number of statistically significant links which are, in general, consistent with the scientific literature.

In the discussion, the authors place emphasis on both the range of behaviours observed among older non-standard workers and the low frequency of the normative behaviour expected at the end-of-career stage, namely disinvolvement from work in favour of life outside work. Based on the life course perspective, this result is seen as an indicator of a destandardization of end-of-career trajectories which, however, might turn out to be a double-edge sword for workers. The diversity of behaviours observed also illustrates the relevance of considering simultaneously life at work and life outside work. As regards the workers' subjective view of their advancing age and the stage of life they are in, attention is drawn to the phenomenon of early psychological disinvolvement from work, which can be interpreted as premature aging, the consequences of which are worrying at individual, organizational and societal levels. Lastly, as regards the link between types of involvement behaviour and the relationship with age and the future, it may be relevant to consider the workers' subjective view of their employment situation, since the latter is moreover dependent on their perception of their career and biographical trajectories. The authors suggest that this association between types of behaviour and the relationship with age and the future can be interpreted as the linkage between individual biographical temporalities (past/present/future). 